\title{
Sleep quality and mood in mothers and fathers accommodated in the family-centred paediatric ward
}

Charlotte Angelhoff, Ulla Edéll-Gustafsson and Evalotte Mörelius

The self-archived postprint version of this journal article is available at Linköping University Institutional Repository (DiVA):

http:// urn.kb.se/ resolve?urn=urn:nbn:se:liu:diva- 143585

N.B.: When citing this work, cite the original publication.

Angelhoff, C., Edéll-Gustafsson, U., Mörelius, E., (2017), Sleep quality and mood in mothers and fathers accommodated in the family-centred paediatric ward, J ournal of Clinical Nursing.

https:// doi.org/ 10.1111/jocn.14092

Original publication available at:

https:// doi.org/ 10.1111/jocn.14092

Copyright: Wiley (12 months)

http:// eu.wiley.com/WileyCDA/ 


\section{Abstract}

Aims and objectives: To describe sleep quality and mood in parents accommodated with their sick child in a family-centred paediatric ward. Secondary aims were to compare mothers' and fathers' sleep quality and mood in the paediatric ward, and to compare the parents' sleep quality and mood between the paediatric ward and in a daily-life home setting after discharge.

Background: Frequent interruptions, ward noise, and anxiety affect parents’ sleep quality and mood negatively when accommodated with their sick child in paediatric wards. Poor sleep quality and negative mood decreases the parents' ability to sustain attention and focus, and to care for their sick child.

Methods: This was a prospective and descriptive study. Eighty-two parents (61 mothers and 21 fathers) with children (median age 6.25 years) admitted to six paediatric wards participated in the study. Uppsala Sleep Inventory, a sleep diary and The MACL were used to measure sleep quality and mood.

Results: The parents had a good sleep quality in the paediatric ward even though they had more nocturnal awakenings compared to home. Moreover, they were less alert, less interested, and had reduced concentration, and were more tired, dull and passive in the hospital than at home after discharge. Vital sign checks, noises made by the staff and medical treatment were given reasons influencing sleep. Poor sleep quality correlated with negative mood.

Conclusion: Parents's sleep quality in family-centred paediatric care is good. However, the habitual sleep efficacy before admittance to the hospital is lower than expected and needs to be further investigated.

Relevance to clinical practice: The healthcare professionals should acknowledge parents' sleep and mood when they are accommodated with their sick child. Further should care at 
night be scheduled and sleep promoted for the parents in order to maintain health and wellbeing in the family.

Key words: Adolescents, Child, Child Nursing, Children’s Nurses, Family-Centred Care, Family Nursing, Hospitalised Child, Paediatrics, Parent, Sleep

What does this paper contribute to the wider global clinical community?

- Parents’ sleep quality was good in family-centred paediatric wards even though they had more nocturnal awakenings compared to home.

- Poor sleep quality in the paediatric ward predicted negative mood, and the parents were less alert, less interested, and had reduced concentration, and were more tired, dull and passive in the hospital than at home after discharge.

- Nurses in paediatric care must acknowledge and promote sleep in parents accommodated with their sick child in hospital. To prevent interrupted sleep for the parents and the child, vital sign checks, procedures and 


\section{INTRODUCTION}

When a child is admitted to hospital, parents should be offered accommodation and be encouraged to stay without additional costs (European Association for Children in Hospital 2001). Family-centred care is a way of providing care for children and their families, ensuring that the whole family is recognized as care recipients (Shields et al. 2006). Based on United Nations Convention of the Rights of the Child, family-centred care with a child-centred approach include the child's perspective and preferences in the planning of the child's care (Coyne et al. 2016). Services should be coordinated around the child's and the family's particular needs, and children, adolescents and parents should be involved as partners in care (Shields 2015). However, paediatric wards are generally designed for the child's medical care, and are not always appropriate for sleep (Stremler et al. 2015). Frequent interruptions, ward noise, and anxiety affect parents' sleep quality negatively (Stremler et al., 2011, Meltzer et al. 2012, Franck et al. 2013) and may decrease the ability to care for their child.

Sleep quality describes how well a person has slept, and how tired they feel on waking. Feeling rested and restored indicates good sleep quality (Harvey et al. 2008). Sleep efficiency (SE\%) refers to the percentage of total time in bed actually spent sleeping, and gives an overall sense of sleep quality. SE\% should be kept steady between 85-89\% (Reed \& Sacco 2015). Individuals experiencing sleep deprivation due to stressful situations are at greater risk of developing persistent insomnia (Jarrin et al. 2014, Yang et al. 2014), which has a negative impact on both psychological (e.g. depression) and physiological (e.g. hypertension, diabetes) health (Morin \& Jarrin 2013). Sleep quality affects working memory (Verweij et al. 2014), the ability to sustain attention and focus, and emotion regulation (Gobin et al. 2015). These are cognitive and emotional abilities that are important for parents of sick children. 
Poor sleep quality due to sleep deprivation is a frequent problem for parents of children with special health-care needs (Mörelius \& Hemmingsson 2014, McBean \& Schlosnagle 2016), leading to forgetfulness, lack of energy, impatience with the family, and negative mood (Angelhoff et al. 2015, McCann et al. 2015). Mood is a subjective description of a persons’ emotional state, determined by disposition and circumstances. It affects self-satisfaction, perception of the world, and behaviour, and is the main feature in the diagnosis of depression (Trzepacz \& Baker 1993).

No previous study to our knowledge, examines parents’ sleep quality and mood in parents resident with their sick child in a non-intensive paediatric care setting, irrespective of the child's age, diagnosis or severity of illness. Therefore, we aimed to describe sleep quality and mood in parents accommodated with their sick child in a family-centred paediatric ward. Secondary aims were to compare mothers' and fathers' sleep quality and mood in the paediatric ward, and to compare the parents' sleep quality and mood between the paediatric ward and in a daily-life home setting after discharge.

\section{METHODS}

\section{Design}

This is a prospective and descriptive study. It is part of a larger project exploring sleep, mood and stress in parents accommodated in paediatric wards. Ethical approval was granted by the Regional Committee for Medical Research (DNR 2011/1631).

\section{Participants}

A convenience sample of parents from six paediatric wards for children (0-18 years) at one university hospital and three general hospitals in south-east Sweden was chosen. Three wards were specialized in oncology/diabetes/neurology, acute/elective surgery, and emergency medicine/infections, respectively. Three wards were general paediatric wards. A sample size 
of 35 parents was calculated on the variable sleep quality to get a statistical power of $80 \%$ $(p<0.05$, effect size Cohen's d 0.25).

\section{Procedure}

Data collection was made in the hospital and four weeks later at home. Based on the protocol from the main study, parents were asked to participate on a Tuesday evening. Inclusion criteria: Swedish-speaking parents staying overnight with a child in the paediatric wards. The parents received oral and written information about the study by the nursing staff on the ward. After signing informed consent, they received an envelope with instructions, questionnaires and a sleep-log. The questionnaires were collected on Wednesday morning by the nursing staff and sent to the first author. The sleep-log was returned by the parents to the first author in a prepaid envelope. Four weeks later, after discharge, the parents received a new sleep-log and questionnaires to complete at home. Data were collected September 2013-October 2015, with intermissions during June-August and December.

\section{Measures}

Demographics: The parents provided data about gender, age, marital status, educational level, the child's age, diagnosis and earlier health status in a study-specific questionnaire.

Habitual sleep patterns before admission: The parents filled out 40 items from the Uppsala Sleep Inventory (USI). USI evaluates perceived sleep during the past four weeks, and provides data on sleep quality (five-point scale; 1 = bad, 5 = good), time going to bed (TTB), sleep onset latency (SOL) (time from light off to falling asleep in minutes), morning awakening time, and sleep duration. The USI has shown good validity and reliability compared to polysomnography (Edell-Gustafson 2002). Cronbach's alpha ( $\alpha$ ) was calculated on 27 items from the USI in this sample. Items measuring time and frequencies were excluded. The internal consistency indicates good reliability $(\alpha=0.89)$. 
Sleep quality: The parents filled out a sleep-log Wednesday morning in the hospital and at home after discharge. The sleep-log provided data on sleep quality (five-point scale; 1 = bad, 5 = good), TTB, SOL, morning awakening time, and nocturnal awakenings (minutes).

Mood: The parents filled out the Mood Adjective Checklist (MACL) Wednesday morning in the hospital and at home after discharge. The MACL consists of 71 adjectives, measured on a 4-point scale ( 1 = it definitely disagrees with what I feel right now, 4 = it definitely agrees with what I feel right now). The adjectives are divided into six dimensions; control (11 items), calmness (10 items), social orientation (11 items), pleasantness (12 items), activation (16 items), and extraversion (11 items) (Sjöberg et al. 1979). The dimensions were analysed separately and as a total mean score with a minimum and maximum of 12 and 47 . Higher values indicate better mood. The dimension of activation (mood-activation) is particularly interesting to study in parents with sick children as it includes emotions such as concentration, interest, alertness, attention, dullness, tiredness, indolence, and passivity. The MACL has shown good validity and reliability in previous studies ( Sjöberg et al. 1979, Persson et al. 1980). In this sample, the internal consistency indicates good reliability in the different dimensions $(\alpha=0.73-0.90)$.

Variables influencing parents' sleep: The sleep-log consisted of one open-ended question about what the parents perceived had influenced their sleep during the night. Several reasons could be stated in the response. As most responses approached as single words, the openended question was analysed with a quantitative content analysis approach. Content analysis is a convenient, scientific method for evaluation of textual types of data such as open-ended questions in questionnaires, and can be applied in both qualitative and quantitative studies. The content analysis of the open-ended question was quantitative, focusing on counting the frequency of specific words or content. The content was identified and quantified, and further analysed to create categories (Kondracki et al. 2002). 


\section{Statistical Analyses}

Frequency $(n)$, percentage $(\%)$, median (md), and quartiles $\left(\mathrm{Q}_{1}, \mathrm{Q}_{3}\right)$ (the $25^{\text {th }}$ and $75^{\text {th }}$ percentiles) were used to describe data. Single missing values (3.5\%) in the MACL were replaced by the sample mean which has been reported as an appropriate method for dealing with missing data when the proportion is $<5 \%$ (Penny \& Atkinsson, 2012). One participant turned the light off 240 minutes after falling asleep. As analysis of SOL assume that the light will be turned off before sleep this single value was removed. However, remaining data from the participant was obtained in the data analyses. Two parents reported that they were not accommodated in single-rooms. As data did not affect the result, they were included in the analyses. The 24-hour clock time notations were converted into decimal hours before analysis. SE\% was calculated as the ratio of sleep duration and TTB times 100.

Wilcoxon Signed Rank Test was used for matched comparisons in sleep variables and mood between the paediatric ward and at home after discharge. Mann Whitney U-test was used to study possible differences in sleep variables and mood between mothers and fathers in the hospital. Spearman's correlation coefficient $(\rho)$ was used to study correlations between sleep variables and mood, and between sleep quality, mood and earlier experience from hospital stay in the paediatric ward.

Stepwise regressions were performed using univariate ANOVA. Sleep quality, nocturnal awakenings, and the total mean score of mood in the paediatric ward were set as the dependent variable in three separate models to study the effect of the independent variables; parents’ gender (categorical) parents’ ages (continuous), children’s ages (continuous), children's diagnosis (categorical; infection, surgery, oncology and other), and the four different hospitals (categorical). 
The statistical software program SPSS version 23 was used for the statistical analyses. $P$ values $<0.05$ were considered statistically significant.

\section{RESULTS}

\section{Demographics and habitual sleep}

The final sample comprised 82 parents. Demographic data of parents and children are provided in Table 1 and 2, respectively. A total of 75 parents (92\%) reported that they had no or few problems falling asleep at night at home before admission. Median score for sleep quality was $3\left(\mathrm{Q}_{1}, \mathrm{Q}_{3}: 2,4\right)$ and sleep duration $6 \mathrm{~h} 30 \mathrm{~min}\left(\mathrm{Q}_{1}, \mathrm{Q}_{3}\right.$ : 6h 0min, 7h 0min). Median score for SE\% was 78.8\% $\left(\mathrm{Q}_{1}, \mathrm{Q}_{3}: 71.0 \%\right.$, 91.2\%). Fifty-six parents (68\%) reported insufficient habitual sleep. Thirty-two parents (39\%) reported frequent nocturnal awakenings, of whom 20 (24\%) woke up due to them worrying about the child.

\section{Parents' sleep quality in the paediatric ward and at home after discharge}

The parents had stayed in the paediatric ward at a median of one night (range: 0-10 nights) when included in the study. All families, except two, had single-patient rooms. Sixty-eight parents (83\%) stayed alone in the room with their child, nine children (11\%) were accompanied by both parents, where of three families choose to stay overnight at the hospital with the whole family, including siblings. In three families both parents participated in the study. A total of 55 parents (67\%) completed the sleep-log. Median score for sleep quality was $3\left(\mathrm{Q}_{1}, \mathrm{Q}_{3}: 2\right.$, 4). Sleep variables are presented in Table 3. Twenty-nine parents (53\%) reported good sleep quality when the rated sleep quality score was dichotomized into good (score 3-5) and poor (score 1-2). Wilcoxon Signed Rank Test showed no significant difference in the matched comparison between habitual sleep quality and sleep quality in the hospital. ANOVA showed no statistically significant effects on sleep quality or nocturnal awakenings of the parent's gender and age, the child's age, diagnosis, or hospital. To control 
for lack of independence (three parent dyads participating from the same family) the ANOVA was recalculated excluding the mothers in those families. This did not affect the result.

Thirty-five parents (43\%) completed the sleep-log at home after discharge. Wilcoxon Signed Rank Test showed that the parents rated their sleep quality significantly higher $(p=0.042)$, and that they reported fewer $(p=0.047)$ nocturnal awakening minutes at home (Table 3). A matched comparison of mothers and fathers separately, showed that the mothers reported more nocturnal awakening minutes $(p=0.045)$ in the paediatric ward compared to at home after discharge, no differences were found in the fathers.

\section{Parents' mood in the paediatric ward and at home after discharge}

Thirty-six parents (44\%) completed the MACL at home after discharge. Matched comparisons were made with Wilcoxon Signed Rank Test on a sample size of 35 parents (Table 4). Mood-activation were significantly lower $(p=0.020)$ in the hospital than at home after discharge. A matched comparison of mothers and fathers separately, showed that mothers had lower mood in total mean score $(p=0.047)$ and in the dimension of activation ( $p$ $=0.032$ ) in the paediatric ward compared to at home after discharge. There was a positive, but weak, correlation between sleep quality and mood total mean-score $(\rho=0.29, p=0.036)$, and between sleep quality and mood activation ( $\rho=0.39, p=0.004$ ), indicating that the poorer the sleep quality, the lower the mood. ANOVA showed no statistically significant effects on the total mean score of mood of the parents' gender and age, the child's age, diagnosis, or hospital.

\section{Comparisons of sleep quality and mood between mothers and fathers}

In the paediatric ward, mothers reported lower $(p=0.012)$ mood in the dimension of control (md: 31, $\mathrm{Q}_{1}, \mathrm{Q}_{2}$ : 27-34) than the fathers (md: 35, $\mathrm{Q}_{1}, \mathrm{Q}_{2}$ : 30-41). No statistically significant differences in the other dimensions of mood or in the sleep variables between mothers and 
fathers were found (Mann Whitney U-test). To control for lack of independence (three parent dyads participating from the same family) the Mann Whitney U-test was recalculated excluding the mothers in those families. The statistical significance remained; the mothers reported lower $(p=0.012)$ mood in the dimension of control (md: 31, $\mathrm{Q}_{1}, \mathrm{Q}_{2}$ : 27-34) than the fathers (md: 35, $\mathrm{Q}_{1}, \mathrm{Q}_{2}$ : 30-41). No significant differences in the other dimensions of mood or in the sleep variables were found.

\section{Parents' perceptions of variables influencing sleep in the paediatric ward}

Six categories explaining variables influencing sleep were detected; the child ( $n=26,46 \%$ ), the staff ( $n=21,38 \%)$, medical treatment ( $n=19,34 \%)$, the environment ( $n=11,20 \%)$, worries ( $n=3,5 \%)$, and that nothing affected the sleep $(n=11,20 \%)$. The main reason for nocturnal awakenings in the hospital was the child. This could be due to the child's normal behaviours, such as nightly awakenings and breastfeeding for small children, but also to coughing or pain, depending on the child's current health. Vital sign checks, noises made by the staff and medical treatment, such as drugs, infusions and blood samples, were other given reasons influencing sleep. At home, most parents ( $n=22,63 \%)$ reported that nothing had influenced their sleep. The given variables had no statistically significant effects on parents' sleep quality.

\section{DISCUSSION}

Having a sick child admitted to hospital requires that the parents are well rested and alert in order to manage the demands of the care setting and maintain the child's health, as well as their own. Few studies describe parents’ sleep quality when they stay at their child's bedside in hospital. This study adds important information about the subject as it provides the first self-reported measures of sleep quality in relation to mood in parents, resident on paediatric wards, irrespective of the child's age, diagnosis, or severity of illness. 
Even though the parents reported more nocturnal awakenings at the hospital compared to home, the parents had a good sleep quality. This is in contrast with previous studies reporting poor sleep quality in parents staying overnight at their child's bedside in hospital (Meltzer et al. 2012, McLoone et al. 2013). The contributing factor to good sleep quality in the present study could be that most families were given accommodation in single-patient rooms, which is becoming standard in most paediatric wards in Sweden, unlike many other countries. In Stremler et al. (2011), parents of children admitted to intensive care struggled with the decision whether to stay at their sick child's bedside or not, due to lack of accommodation possibilities. This affected their sleep negatively and led to feelings of guilt and worries. In Meltzer et al. (2012), 43\% of the families shared a room with another patient and reported that room-mates making noise interfered with sleep. Single-patient rooms are reported as the only variable that significantly influences parental sleep duration in hospital (McCann 2008). Parents in a qualitative study have expressed how being together in in a private room improved sleep and time for relaxation (Edell-Gustafsson et al. 2015).

Our results show that poor sleep quality in the paediatric ward is associated with negative mood, even though the correlations were weak. The connection between sleep quality and mood has been reported earlier in healthy adults (Finan et al. 2015). Negative mood may not only affect the parents’ ability to care for their child and make decisions regarding their child's care, but may also lead to depression, which further increases the risk for chronic sleep problems (Morin \& Jarrin 2013). In the present study, there was a difference in moodactivation between the paediatric ward and at home after discharge, indicating that the parents were less alert, less interested, and had reduced concentration, and were more tired, dull and passive in the hospital. Mothers also reported lower scores in the dimension of control, indicating that they felt insecure and inhibited in the paediatric ward. However, children's diagnoses had no effect on the parents’ sleep quality or mood. 
According to Morin et al. (2003), it is the way a person perceives a stressful situation, rather than the actual stress itself, that causes sleep deprivation. Picoraro et al. (2014) reported that parents of seriously ill children often find ways to endure the situation and even learn to benefit from the experience, including greater appreciation of life, greater personal strength, and recognition of new possibilities. Coping was not studied in this study, but the importance of coping ability in relation to parental stress has been reported in previous studies (VrijmoetWiersma et al. 2009, Muscara et al. 2015). Parents with more efficient coping skills may adapt to the situation and therefore decrease bedtime arousal and maintain a good sleep quality, despite the stressful situation.

It is important to highlight that the parents had a low SE\% (<85\%) already at home before the hospital stay. This indicates that the parents' sleep quality was poor four weeks before their child was admitted to the hospital. Low SE\% has previously been described in parents of children with cancer as well as in parents of healthy children (McLoone et al. 2013). It is important for the health-care professionals in primary health-care and in school health-care to ask parents about their sleep, as well as their child's, in order to prevent future health-related problems. Health-care professionals need tools to identify parents at risk of developing sleep deprivation and recommend interventions in order to improve sleep quality and thus maintain health and well-being in the family.

The strength of this study is that parents were included from six different paediatric wards, irrespective of their child's age, diagnosis or severity of illness. The prospective design was chosen to enable repeated data collection from the same individuals, making it possible to evaluate potential changes in sleep quality and mood between staying at the hospital and at home after discharge. However, the convenience sampling in this study has its limitations. It was difficult to enrol parents because their children were the primary focus of their hospital admission. A selection bias could be that the participating parents were the most alert parents, 
and that those who were tired declined participation in the study. Therefore, this sample may not be a representative sample for all parents accommodated with their sick child in paediatric wards. Nevertheless, the results are important as this is the first study of sleep and mood in both mothers and fathers of children in all ages, with different severity of illness.

Another limitation could be that it was impossible to compare the views of parents in family centred care settings with parents who were not in care settings without a family centred care philosophy for ethical reasons as family centred care was the standard care philosophy on the paediatric wards in this study.

All data was provided by self-reported measurements. Future research should complement these subjective measures of sleep with objective measures, such as cortisol awakening response and actigraphy. Furthermore, a qualitative interview study is justified to obtain a deeper understanding of parents' sleep quality, mood and coping strategies when they stay with their child in a paediatric ward, and to find more underlying causes influencing sleep quality.

\section{Conclusion}

Parents report good sleep quality when they stay in the paediatric ward with their child even though it improves at home after discharge. However, frequent interruptions during the night in hospital hinder sleep, and the parents' mood is lower in the morning after the night in hospital than at home after discharge. No differences were found in the sleep variables between mothers and fathers, however mothers had lower mood in the dimension of control at the hospital. Our results support the importance of family-centred care and single-patient rooms where the healthcare professionals acknowledge parents' sleep and mood, schedule care at night, and promote sleep for the parents in order to maintain health and well-being in the family. 


\section{Relevance to clinical practice}

Nurses in paediatric care should be encouraged to acknowledge signs of parental sleep deprivation and negative mood through information and education about the importance of good parental sleep quality, as this can affect the child's health. At the hospital, the necessity to go to the patient's room at night should be considered, although patient safety must always be prioritised. We suggest that vital sign checks, procedures and medical treatments should be scheduled at the same time when possible, to prevent interrupted sleep for the parents and the child. Furthermore, health care professionals should encourage and provide opportunities for the parents to take turns during extended hospital stays in order to provide a change of scenery and for a chance to regain lost sleep.

\section{Acknowledgements}

We would like to acknowledge the participating parents in the study, the nursing staff at the paediatric wards for logistical support.

\section{References}

Angelhoff C, Edéll-Gustafsson U \& Mörelius E (2015): Sleep of Parents Living With a Child Receiving Hospital-Based Home Care: A Phenomenographical Study. Nursing research 64, 372-380. doi: 10.1097/NNR.0000000000000108.

Coyne I, Hallström I \& Söderbäck M (2016): Reframing the focus from a family-centred to a child-centred care approach for children's healthcare. Journal of child health care. doi: $10.1177 / 1367493516642744$.

Edéll-Gustafsson UM (2002): Insufficient sleep, cognitive anxiety and health transition in men with coronary artery disease: a self-report and polysomnographic study. Journal of Advanced Nursing 37, 414-422. 
Edéll-Gustafsson UM, Angelhoff C \& Mörelius E (2015) Hindering and buffering factors for sleep in neonatal care. A phenomenographic study. Journal of Clinical Nursing 24(56), 717-27. doi: 10.1111/jocn.12654.

European Association for Children in Hospital (2001) The 10 articles of the EACH charter. https://www.each-for-sick-children.org/each-charter/the-10-articles-of-the-eachcharter.html 2016-07-06.

Finan PH, Quartana PJ \& Smith MT (2015): The Effects of Sleep Continuity Disruption on Positive Mood and Sleep Architecture in Healthy Adults. Sleep 38, 1735-1742. doi: 10.5665/sleep.5154.

Franck LS, Gay CL \& Rubin N (2013): Accommodating families during a child's hospital stay: Implications for family experience and perceptions of outcomes. Families, Systems and Health 31, 294-306. doi: 10.1037/a0033556.

Gobin CM, Banks JB, Fins AI \& Tartar JL (2015): Poor sleep quality is associated with a negative cognitive bias and decreased sustained attention. Journal of Sleep Research 24, 535-542. doi: 10.1111/jsr.12302.

Harvey AG, Stinson K, Whitaker KL, Moskovitz D \& Virk H (2008): The subjective meaning of sleep quality: a comparison of individuals with and without insomnia. Sleep 31, 383-393.

Jarrin DC, Chen IY, Ivers H \& Morin CM (2014): The role of vulnerability in stress-related insomnia, social support and coping styles on incidence and persistence of insomnia. Journal of Sleep Research 23, 681-688. doi: 10.1111/jsr.12172.

Kondracki NL, Wellman NS \& Amundson DR (2002): Content analysis: review of methods and their applications in nutrition education. Journal of nutrition education and behavior 34, 224-230. 
McBean AL \& Schlosnagle L (2016): Sleep, health and memory: comparing parents of typically developing children and parents of children with special health-care needs. Journal of Sleep Research. 25(1), 78-87. doi: 10.1111/jsr.12329

McCann D (2008): Sleep deprivation is an additional stress for parents staying in hospital. Journal for specialists in pediatric nursing. 13, 111-122. doi: 10.1111/j.17446155.2008.00142.x.

McCann D, Bull R \& Winzenberg T (2015): Sleep Deprivation in Parents Caring for Children With Complex Needs at Home: A Mixed Methods Systematic Review. Journal of Family Nursing 21, 86-118. doi: 10.1177/1074840714562026

McLoone JK, Wakefield CE, Yoong SL \& Cohn RJ (2013): Parental sleep experiences on the pediatric oncology ward. Supportive care in cancer 21, 557-564. doi: 10.1007/s00520012-1547-z.

Meltzer7 LJ, Davis KF \& Mindell JA (2012): Patient and parent sleep in a children's hospital. Pediatric nursing 38, 64-71; quiz 72.

Mörelius E \& Hemmingsson H (2014): Parents of children with physical disabilities perceived health in parents related to the child's sleep problems and need for attention at night. Child: Care, Health and Development 40, 412-418. doi: 10.1111/cch.12079.

Morin CM \& Jarrin DC (2013): Epidemiology of insomnia: Prevalence, course, risk factors, and public health burden. Sleep Medicine Clinics 8, 281-297. doi: 10.1016/j.jsmc.2013.05.002

Morin CM, Rodrigue S \& Ivers H (2003): Role of stress, arousal, and coping skills in primary insomnia. Psychosomatic medicine 65, 259-267.

Muscara F, McCarthy MC, Woolf C, Hearps SJ, Burke K \& Anderson VA (2015): Early psychological reactions in parents of children with a life threatening illness within a 
pediatric hospital setting. European psychiatry 30, 555-561. doi:

10.1016/j.eurpsy.2014.12.008.

Persson LO, Sjöberg L \& Svensson E (1980): Mood effects of alcohol. Psychopharmacology 68, 295-299.

Penny KI \& Atkinsson I (2012): Approaches for dealing with missing data in health care studies. Journal of Clinical Nursing 21, 2722-2729. doi: 10.1111/j.1365-2702-201103854.x

Picoraro JA, Womer JW, Kazak AE \& Feudtner C (2014): Posttraumatic growth in parents and pediatric patients. Journal of palliative medicine 17, 209-218. doi: 10.1089/jpm.2013.0280.

Reed DL \& Sacco WP (2015): Measuring Sleep Efficiency: What Should the Denominator Be? Journal of clinical sleep medicine 12, 263-266. doi: 10.5664/jcsm.5498.

Shields L (2015): What is "family-centered care"? European Journal for Person Centered Healthcare 3, 139-144. doi: 10.5750/ejpch.v3i2.993

Shields L, Pratt J \& Hunter J (2006): Family centred care: A review of qualitative studies. Journal of Clinical Nursing 15, 1317-1323. doi: 10.1111/j.1365-2702.2006.01433.x

Sjöberg L, Svensson E \& Persson LO (1979): The measurement of mood. Scandinavian Journal of Psychology 20, 1-18.

Stremler R, Adams S \& Dryden-Palmer K (2015): Nurses' Views of Factors Affecting Sleep for Hospitalized Children and Their Families: A Focus Group Study. Research in nursing \& health 38(4), 311-322. doi: 10.1002/nur.21664

Stremler R, Dhukai Z, Wong L \& Parshuram C (2011): Factors influencing sleep for parents of critically ill hospitalised children: a qualitative analysis. Intensive \& critical care nursing 27, 37-45. doi: 10.1016/j.iccn.2010.11.001. 
Trzepacz PT \& Baker RW (1993) Mood and Affect. In The Psychiatric Mental Status Examination (Trzepacz PT \& Baker RW eds.). Oxford University Press, Inc., New York, p. 39.

Verweij IM, Romeijn N, Smit DJ, Piantoni G, Van Someren EJ \& van der Werf YD (2014): Sleep deprivation leads to a loss of functional connectivity in frontal brain regions. BMC neuroscience 15, 88-2202-2215-2288. doi: 10.1186/1471-2202-15-88.

Vrijmoet-Wiersma CMJ, Ottenkamp J, Van Roozendaal M, Grootenhuis MA \& Koopman HM (2009): A multicentric study of disease-related stress, and perceived vulnerability, in parents of children with congenital cardiac disease. Cardiology in the Young 19, 608-614. doi: 10.1017/S1047951109991831.

Yang C, Hung C \& Lee H (2014): Stress-related sleep vulnerability and maladaptive sleep beliefs predict insomnia at long-term follow-up. Journal of Clinical Sleep Medicine 10, 997-1001. doi: $10.5664 /$ jcsm.4036. 
Table 1. Parent demographics

$n=82$

\begin{tabular}{lr} 
Age in years, median $\left(\mathrm{Q}_{1}, \mathrm{Q}_{3}\right)$ & $37(32,44)$ \\
Gender & \\
Mother & $61(74.4 \%)$ \\
Father & $21(25.6 \%)$ \\
Education & $2(2.4 \%)$ \\
Compulsory school & $28(34.1 \%)$ \\
Upper secondary school & $43(52.4 \%)$ \\
University & $4(4.9 \%)$ \\
Other & $5(6.1 \%)$ \\
Missing data & \\
Marital status & $71(86.6 \%)$ \\
Married/cohabitant & $5(6.1 \%)$ \\
Single & $1(1.2 \%)$ \\
In a relation, separate living & $5(6.1 \%)$ \\
Missing data & $12(14.6 \%)$ \\
Nicotine use & \\
Been to the hospital with the & \\
child before & $16(19.5 \%)$ \\
Never & $13(15.9 \%)$ \\
Once & $22(26.8 \%)$ \\
2-5 times & $10(12.2 \%)$ \\
6-10 times & $18(22.0 \%)$ \\
>10 times & $3(3.7 \%)$ \\
Missing data & \\
\hline
\end{tabular}


Table 2. Child demographics

$n=79$

The child's age in years, median $\left(Q_{1}, Q_{3}\right)$

$6.25(1.5,13.0)$

The child's diagnosis, frequency

Infection

$31(39.2 \%)$

Surgery (acute/elective)

$23(29.1 \%)$

Oncology

$9(11.4 \%)$

Other

$13(16.5 \%)$

Missing data 
Table 3. Parents' sleep in the paediatric ward and at home after discharge

\begin{tabular}{|c|c|c|c|}
\hline & $\begin{array}{l}\text { Sleep in the } \\
\text { paediatric ward } \\
n=35 \\
\text { median }\left(Q_{1}, Q_{3}\right)\end{array}$ & $\begin{array}{l}\text { Sleep at home } \\
\text { after discharge } \\
n=35 \\
\text { median }\left(Q_{1}, Q_{3}\right)\end{array}$ & $p$-value ${ }^{a}$ \\
\hline Time to bed (hh:mm) & $22: 30(21: 30,23: 00)$ & $22: 30(22: 00,23: 10)$ & .837 \\
\hline $\begin{array}{l}\text { Sleep onset latency } \\
\text { (minutes) }\end{array}$ & $15(10,35)$ & $10(10,15)$ & .068 \\
\hline $\begin{array}{l}\text { Morning awakening time } \\
\text { (hh:mm) }\end{array}$ & $6: 45(6: 00,7: 25)$ & $6: 15(5: 20,7: 00)$ & .100 \\
\hline $\begin{array}{l}\text { Nocturnal awakenings } \\
\text { (minutes) }\end{array}$ & $20(10,60)$ & $5(0,30)$ & $.047^{*}$ \\
\hline Sleep duration & $\begin{array}{l}\text { 6h 55min } \\
\text { (6h } 11 \mathrm{~min}, 8 \mathrm{~h} \mathrm{0min)}\end{array}$ & $\begin{array}{l}\text { 7h } 5 \min \\
\text { (6h } 5 \mathrm{~min}, 7 \mathrm{~h} 50 \mathrm{~min})\end{array}$ & .908 \\
\hline Sleep quality & $3(2,4)$ & $4(3,5)$ & $.042 *$ \\
\hline Sleep efficiency & $82.9 \%(72.1 \%, 89.9 \%)$ & $85.8 \%(77.2 \%, 92.5 \%)$ & .466 \\
\hline
\end{tabular}

a) Wilcoxon Signed Rank Test ${ }^{*}$ significant at $p<0.05$ 
Table 4. Parents' mood in the paediatric ward and at home after discharge

\begin{tabular}{llll}
\hline & $\begin{array}{l}\text { Mood in the } \\
\text { paediatric ward } \\
n=35\end{array}$ & $\begin{array}{l}\text { Mood at home } \\
\text { after discharge } \\
\text { median }\left(\mathrm{Q}_{1}, \mathrm{Q}_{3}\right)\end{array}$ & $\begin{array}{l}\boldsymbol{p}^{\text {-value }} \mathrm{a}^{\mathrm{a}} \\
\text { median }\left(\mathrm{Q}_{1}, \mathrm{Q}_{3}\right)\end{array}$ \\
\hline Control & $33(27,36)$ & $36(32,38)$ & .421 \\
Calmness & $27(22,32)$ & $31(26,36)$ & .185 \\
Social orientation & $35(30,39)$ & $36(33.40)$ & .797 \\
Pleasantness & $33(27,39)$ & $38(35,43)$ & .141 \\
Activation & $41(36,48)$ & $48(43,54)$ & $.021^{*}$ \\
Extraversion & $31(25,36)$ & $34(28,39)$ & .093 \\
Total mean score & $33(28,37)$ & $37(33,41)$ & .073 \\
\hline
\end{tabular}

a) Wilcoxon Signed Rank Test ${ }^{*}$ significant at $p<0.05$ 